\title{
Efficient numerical integrator based on Fer expansion: Application to solid-state NMR experiments and to solve quantum Liouville equation and quantum Fokker-Planck equation
}

\author{
Eugene Stephane Mananga ${ }^{1,2,3^{*}}$ \\ ${ }^{1}$ PH.D Program Physics \& PH.D Program Chemistry, The Graduate Center, The City University of New York, \\ 365 Fifth Avenue, New York, NY, 10016, USA \\ emananga@gradcenter.cuny.edu \\ ${ }^{2}$ The City University of New York, BCC, Department of Engineering, Physics, and Technology, 2155 \\ University Avenue, CPH 118, Bronx, New York 10453, USA \\ eugene.mananga@bcc.cuny.edu \\ ${ }^{3}$ Department of Applied Physics, New York University, 6 Metrotech Center, Brooklyn, NY 11201, USA \\ eugene.mananga@nyu.edu
}

\begin{abstract}
There are two important related research areas that I propose to investigate. First, we plan to develop an efficient numerical integrator based on Fer expansion for solid-state NMR simulation of experiments. Second, we intend to extend the method to solve quantum Liouville equation and quantum Fokker-Planck equation in order to improve the understanding of the dynamics of quantum systems subject to dissipation due to its relation to macroscopic quantum phenomena. The goal of the proposed research is to study a numerical integrator based on Fer expansion (Fer integrators of higher orders) in the integration of the time-dependent Schrodinger equation (TDSE) which is a central problem to nuclear magnetic resonance in general and solid-state NMR (SSNMR) in particular. The Fer integrator will provide to experts in quantum mechanics, NMR spectroscopy, and spin dynamics researchers, additional means for controlling spin dynamics in SSNMR. The efficient diagram will be used to compare the different orders of the Fer integrators obtained.
\end{abstract}

\section{Key Words}

Solid-state NMR; Fer expansion; Fer numerical integrator; quantum Liouville equation; quantum Fokker-Planck equation;

\section{INTRODUCTION}

The goal of the proposed research is to study a numerical integrator based on Fer expansion (Fer integrators of higher orders) in the integration of the time-dependent Schrodinger equation (TDSE) which is a central problem to nuclear magnetic resonance in general and solid-state NMR (SSNMR) in particular. The Fer integrator will provide to experts in quantum mechanics, NMR spectroscopy, and spin dynamics researchers, additional means for controlling spin dynamics in SSNMR. The efficient diagram will be used to compare the different orders of the Fer integrators obtained. Solving the Schrodinger equation towards obtaining propagators is a central problem to NMR in general and solid-state NMR in particular. The evolution operator allows obtaining the density matrix of the spin system that has evolved from the equilibrium density matrix due to the RF irradiation. Simulations are based on the propagation of the density matrix and only closed form exist for time-independent or self-commuting Hamiltonian. Driven by demands of the experimental NMR, numerous computer programs including SPINACH ${ }^{1}$ (based on Matlab), SIMPSON ${ }^{2}$ (based on scripting interface, Tcl), and SPINEVOLUTION ${ }^{3}$ (based on Chebyshev polynomial expansion), have been developed over the past few decades for the simulation of NMR experiments. Numerical simulations of NMR experiments are often required for the development of new techniques and for the extraction of structural and dynamic information from the spectra.

The applications of the Fokker-Planck equation ${ }^{4}$ to solid-state NMR are considered. I intend to extend the method to solve quantum Liouville equation ${ }^{5}$ and quantum Fokker-Planck equation in order to improve the understanding of the dynamics of quantum systems subject to dissipation due to its relation to macroscopic quantum phenomena.

\section{Significance}

The development of efficient numerical integrator based on Fer expansion for solid-state NMR simulation of experiments is essential. Numerical methods for differential equations are one of the notable glories of contemporary science. Coupled with much algorithmic ingenuity, incorporating careful error control and powerful methods for linear algebraic computations, numerical methods are widely applied across science and engineering fields. One of the most important numerical methods is the numerical integration. It has been the focus of intense research since its development in 1915 by David Gibb ${ }^{6}$. Numerical integration forms a broad family of algorithms for computing the numerical value of a definite integral. Also called numerical quadrature, numerical integration can be used to delineate the numerical solution of differential equations ${ }^{7}$. There has been great interest in solving the celebrated Schrodinger equation, which is a partial differential equation in quantum mechanics that describes how the state of a quantum system modifies with time. Solving the Schrodinger equation towards obtaining propagators is a central problem to NMR in general and also solid-state NMR in particular ${ }^{8-15}$. 
Over the last half century, several theories have been developed and introduced to NMR. Among these approaches are average Hamiltonian theory (AHT) ${ }^{16-21}$ based on Magnus expansion ${ }^{21}$ and Floquet theory (FLT) $)^{22-24}$ based on van Vleck perturbation $^{25,26}$. These theories have been successful for designing sophisticated pulse sequences and the understanding of different experiences. A major setback to the greater applicability of AHT and FLT is limited to the stroboscopic detection scheme and to the simplification of calculations of "the points in between", respectively. This had recently led us to introduce the fusion of AHT and FLT called Floquet-Magnus expansion (FME) ${ }^{8}$ to solid-state NMR. The main advantage of the FME is that the unitary character of the evolution operator is preserved at all orders of approximation. Even so, the drawback of the scheme (FME) is that the approach is divergent in general. Theoretical approaches are useful and powerful tools for more accurate and efficient spin dynamics simulation to understand experiments and devising new RF pulse sequence in NMR. Solid-state NMR is an important method of spectroscopy used to study structure, dynamic properties of solid materials, kinetics and thermodynamics of a variety of systems ${ }^{27-30}$.

Another emerging theory in the field of solid-state NMR is called Fer expansion (FE) ${ }^{31}$ and is the purpose of this proposal. The Fer expansion was formulated by Fer in 1958 and later revised by Fer, Klarsfeld and Oteo, Casas, Blanes and coworkers $^{32,33}$. The FE approach was recently introduced to the NMR community by Madhu and Kurur in 2006 via the effect of Bloch-Siegert shift and heteronuclear dipolar decoupling ${ }^{34,35}$. While the Magnus expansion and Floquet theory are common in solid-state NMR field, the Fer method is a relatively new comer although the mathematical formalism has been known for several decades. A recent comparison among the above approaches (AHT, FLT, FME, and FE) reported by Takegoshi and co-workers in solid-state NMR indicated that the Fer expansion is more suitable for examination of solidstate NMR experiments ${ }^{36,37}$. Furthermore, the Fer expansion presents advantages over other approaches such as a possibility of longer timestep ${ }^{35}$. For example, if the numerical model is implemented with the approximate solutions of Magnus, the results of the simulation will show incorrect or undesirable effects of finite pulses and ring-down mainly when dealing with quadrupolar nuclei $(\mid>1 / 2)$. The question is how fast will the quadrupolar Hamiltonian evolves during the timestep? Current state-of-the art allows very fast spinning $(>100 \mathrm{kHz})$ so that a much smaller timestep may be required to be in the situation where the rotation during the timestep is neglected. Standard approach adopted in the NMR community is to make the timestep small on which the Hamiltonian is assumed to be time-independent.

\section{Objective}

The overall goals are:

a) to develop Fer integrators of higher orders;

b) to use the efficient diagram to compare the different orders of the Fer integrators obtained, for use in solid-state NMR;

c) the efficiency plot is obtained by carrying out the numerical integration with different time steps, corresponding to different numbers of evaluations of the Hamiltonian $\mathrm{H}(\mathrm{t})$ (For each run, one compares the corresponding approximation with the exact solution, and plots the error as a function of the total number of matrix evaluations).

d) to apply the efficient numerical integrator based on Fer expansion to the following important solid-state NMR experiments such as: spin locking, Triple Oscillating Field Technique (TOFU), three level systems, and TwoPulse Phase-Modulated (TPPM) decoupling.

e) to attract new researchers into the field of spin dynamics. To achieve these goals, the integrated scientific and educational objectives of this proposal are: 1$)$ the development of higher orders $\left(10^{\text {th }}, 12^{\text {th }}\right.$, etc $\left.\ldots ..\right)$ Fer integrators methods; 2) the use of the Fer integrator with best performance to solve the TDSE in order to obtain the appropriated propagators. The evolution operator allows to obtain the density matrix of the spin system that has evolved from the equilibrium density matrix due to the application of RF irradiation. The density matrix at all time during the experiment is needed to have the signal as a function of the time, $t$.

\section{Research Background and Technical Approach for Achieving Research Objective}

The question that arises is: how to develop an efficient Fer integrator of high order and implement it in spin dynamics simulation of solid-state NMR experiment? The Fer based methods, have been divided into two subfamilies, Fer and symmetric $\mathrm{Fer}^{35}$. This is based on their arguments of the exponential in terms of the successive approximations. An essential propriety for TDSE is that the Fer expansion retains its full order of convergence if the exact solution is sufficiently regular. For simplicity, we consider a constant time step, $h=t / N$, with $t_{j}=j h, j=0,1,2 \ldots, N$, and we compute approximations $y_{j}$ to the exact values $Y\left(t_{j}\right)$. To obtain $y_{j}$ we apply the Fer expansion in each subinterval $\left\lfloor t_{j-1}, t_{j}\right\rfloor, j=0,1,2 \ldots, N$, to the initial condition $y_{j-1}$. The process involves three steps. First, the expansions are truncated according to the order in $\boldsymbol{h}$ we want to achieve. Second, the multivariate integrals in the truncated expansions are replaced by conveniently chosen approximations. Third, the exponentials of the matrices have to be computed. We will use the Mathematica software as an efficient tool to compute the matrix exponential or its action on a vector. An analysis of the Fer expansion shows that $F_{k}(h)=O\left(h^{2^{k}-1}\right), k=1,2, \ldots$ and so for instance, in the 
recursive scheme of Fer expansion, $F_{1}, F_{2}$ suffice to build methods up to order six in $h$. Next, using appropriate quadrature rules, one has to approximate the integrals from the recursive scheme of Fer expansion. It turns out that the structure allows one to approximate all the multivariate integrals up to a given order just by evaluating the time dependent Hamiltonian $H(t)$ at the nodes of a univariate quadrature, and this can be done in many different ways. A procedure to obtain methods which can be easily adapted for different quadrature rules uses the averaged matrices. As applications, we will use the most efficient Fer numerical integrator to solve the TDSE to obtain the effective propagator that continually improves the detected NMR signal. We plan to apply the efficient numerical integrator based on Fer expansion to the following specific solid-state NMR experiments which are of major interest in the NMR community. The evolution operator allows obtaining the density matrix of the spin system that has evolved from the equilibrium density matrix due to the RF irradiation. Simulations are based on the propagation of the density matrix and only closed form exists for timeindependent or self-commuting Hamiltonian. We will also compare the performance of the most efficient numerical integrator based on Fer expansion with respect to other Lie-group solvers, namely Magnus and Cayley methods ${ }^{35}$. It is important to mention that a lot of work has been done in partial differential equation mathematical field by authors such as Blanes, Casas, Oteo et al. that has not yet been used in NMR field.

\section{Extension the Fer numerical integrator to solve quantum Liouville equation and quantum Fokker-Planck equation.}

Quantum Liouville equation ${ }^{4}$ : The Liouville equation is a fundamental equation of statistical mechanics which provides a complete description of the system both at equilibrium and away from equilibrium. Its analog in quantum mechanics called quantum Liouville equation describes the time evolution of a mixed state. In quantum systems, the state of a system is defined in terms of a density matrix. We plan to use the Fer numerical integrator to solve the quantum Liouville equation with the density operator having a matrix representation in a basis set.

Quantum Fokker-Planck equation ${ }^{5}$ : The dynamics of quantum systems subject to dissipation is a topic of fundamental importance which has been of major interest for nearly half a century due to its relation to macroscopic quantum phenomena. The quantum Fokker-Planck equation describes such systems. We propose in this proposal to implement the Fer numerical integrator as an efficient theoretical approach to solve quantum Fokker-Planck equation which governs larger systems.

The question that arises is: how an efficient numerical integrator based on Fer expansion can be used to solve quantum Liouville equation and quantum Fokker-Planck equation?

\section{Expected Outcomes}

I plan to obtain the evolution operator by studying the Fer expansion as numerical integrator (Fer integrators of higher orders) that solves the time dependent Schrodinger equation in NMR. The evolution operator allows obtaining the density matrix of the spin system that has evolved from the equilibrium density matrix due to the RF irradiation. Simulations are based on the propagation of the density matrix and only closed form exist for time-independent or self-commuting Hamiltonian. This project is unique because its long term goal is to develop an Advanced Spin Evolution program which will serve all aspects of solid-state NMR simulations for large spin systems. As already mentioned above, numerous computer programs including SPINACH (based on Matlab), SIMPSON (based on scripting interface, Tcl), and SPINEVOLUTION (based on Chebyshev polynomial expansion), have been developed over the past few decades for the simulation of NMR experiments. But, unlike the previous programs, this Advanced Spin Evolution program will rely on the Fer expansion as numerical integrator for the computational technique responsible for the high efficiency of multi-spin computations in which sense the present study is an addition to the current software and programs available in NMR community.

The intention of this proposal is to help bring the current and future prospective theoretical aspects of spin dynamics to the attention of the NMR community and lead new interactions between NMR experts and other specialists in related fields. All of these points strongly support the idea that numerical integrators approaches can also be very useful and powerful tools in quantum spin dynamics.

\section{Reference}

1. Hogben, H. J., Krzystyniak, M., Charnock, G. T. P., Hore, P. J., and Kuprov, I. 2011, Spinach--a software library for simulation of spin dynamics in large spin systems.

2. Bak, M., Rasmussen, J. T., Nielsen, N. C. 2000. SIMPSON: a general simulation program for solid-state NMR spectroscopy.

3. Veshtort, M., Griffin, R. G., 2006. SPINEVOLUTION: A powerful tool for the simulation of solid and liquid state NMR experiments.

4. Narcowich, F. J., 1985. Physica A: Statistical Mechanics and its Applications.

5. Chang, L. D., Wasman, D., 1985. Quantum Fokker-Planck equation.

6. Gibb, D., 1915. A course in interpolation and numerical integration for the mathematical laboratory. 
7. Stoer, J., R. Bulirsch, 1980. Introduction to Numerical Analysis

8. Mananga, E. S. and Charpentier, T., 2011. Introduction of the Floquet-Magnus expansion in solid-state nuclear magnetic resonance spectroscopy.

9. Mananga, E. S., and Charpentier, T., 2016. On the Floquet-Magnus expansion: Applications in solid-state nuclear magnetic resonance and physics.

10. Mananga, E. S., and Charpentier, T., 2015. Floquet-Magnus expansion for general N-coupled spins systems in magic-angle spinning nuclear magnetic resonance spectra.

11. Mananga, E. S., Reid A. E. and T. Charpentier, 2015. Efficient theory of dipolar recoupling in solid-state nuclear magnetic resonance of rotating solids using Floquet-Magnus expansion: Application on BABA and C7 radiofrequency pulse sequences.

12. Evans, W. 1968. Spectroscopy of Condensed Media: Dynamics of Molecular Interactions.

13. Haeberlen, U. 1978. Approach to High-Resolution nmr in Solids.

14. Fel'dman, E. B. 1984. On the convergence of the Magnus expansion for spin systems in periodic magnetic fields.

15. Mananga, E. S., C. D. Hsu, S. Ishmael, T. Islam, G. S. Boutis. 2008. Probing the validity of average Hamiltonian theory for spin $I=1,3 / 2$ and $5 / 2$ nuclei by analyzing a simple two-pulse sequence.

16. Haeberlen, U., and J. S. Waugh, 1968. Coherent Averaging Effects in Magnetic Resonance.

17. Evans, W. 1968. On some applications of the Magnus expansion in nuclear magnetic resonance.

18. Vandersypen, L. M. K., Chuang, I. L. 2004 NMR techniques for quantum control and computation.

19. Fel'dman, E. B., Hitrin, A. K., and Provotorov, B. N. 1983. On the equivalence of different effective hamiltonians which determine the dynamics of a spin system in rapidly oscillating periodic fields.

20. Fel'dman, E. B 1984. Thermodynamics of spin systems in periodic magnetic fields.

21. Magnus, W. 1954. On the exponential solution of differential equations for a linear operator.

22. Floquet, M. G., Ann. Econ. Norm. Suppl. 12, 47 (1883).

23. Shirley, J. H. 1965. Solution of the Schrödinger Equation with a Hamiltonian Periodic in Time.

24. Zur, Y., Levitt, M. H., and Vega, S. Multiphoton NMR spectroscopy on a spin system with I = 1/2.

25. Goldman, M., Grandinetti, P. J., Llor, A., Olejniczak, Z., Sachleben, J. R., and Zwanziger, J. W. 1992. Theoretical Aspects of Higher-Order Truncations in Solid-State NMR.

26. Van Vleck, J. H. 1929. The Effect of Second Order Zeeman Terms on Magnetic Susceptibilities: Errata.

27. Levitt, M. H. 2001. Spin Dynamics: Basics of Nuclear Magnetic Resonance, Wiley.

28. Mehring, M. 1983. Principles of High Resolution NMR in Solids, 2nd Ed.

29. Slichter, C. P., 1990. Principles of Magnetic Resonance, 3rd Ed.

30. Ramamoorthy, A., 2006. NMR Spectroscopy of Biological Solids, Taylor \& Francis, New York.

31. Fer, F., 1958. Resolution de l'equation matricielle $\mathbf{U}=\mathrm{pU}$ par produit infini d'exponentielles matricielles.

32. Casas, F., Oteo, J. A., Ros, J., 1991. Lie algebraic approach to Fer's expansion for classical Hamiltonian systems.

33. Blanes, S., Casas, F., Oteo, J. A., Ros, 1998. Magnus and Fer expansions for matrix differential equations: the convergence problem

34. Madhu, P. K., Kurur, N. D., 2006. Fer expansion for effective propagators and Hamiltonians in NMR.

35. Mananga, E. S., 2016. On the Fer expansion: Applications in solid-state nuclear magnetic resonance and physics.

36. Mananga, E. S., 2013. Applications of Floquet-Magnus expansion, average Hamiltonian theory and Fer expansion to study interactions in solid state NMR when irradiated with the magic-echo sequence.

37. Takegoshi, K., Miyazawa, N., Sharma, K., and Madhu, P. K., 2015. Comparison among Magnus/Floquet/Fer expansion schemes in solid-state NMR. 


\section{Biography}

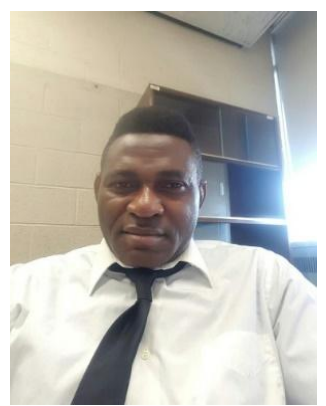

Dr. Eugene Mananga is a Faculty Member in the Physics Doctorate Program and in the PH. D Program in Chemistry at the Graduate Center of the City University of New York. He is an Assistant Professor of Physics and Nuclear Medicine at BCC of CUNY, and an Adjunct Professor of Applied Physics at New York University. He completed his PH.D in Physics from the Graduate Center of the City University of New York, and holds 6 additional graduate degrees and training from various institutions including Harvard University (HMS), Massachusetts General Hospital $(\mathrm{MGH})$, and City College of New York (CCNY). Eugene did his postdoctoral studies in the National High Magnetic Field Laboratory of USA, Harvard Medical School, and Massachusetts General Hospital. Prior to joining Harvard - MGH, Eugene Mananga was an "Ingenieur de Recherche" in the French Atomic Energy Commission and Alternative Energies (CEA-SACLAYNEUROSPIN). Eugene has published more than 40 peer-review scientific articles including prestigious top scientific journals such as Physics Reports, Royal Society of Chemistry (RSC), the Journal of Chemical Physics (JCP), the Journal of Physical Chemistry A and B (JPC A,B), Chemical Physics (CP), etc... and has been serving as editorial board member for more than 20 remarkable journals. He currently serves as Editor-in-Chief of the Journal of Imaging Science (JIS). Dr. Eugene Mananga scientific contribution in the field of Nuclear Magnetic Resonance was honored during the $70^{\text {th }}$ anniversary $(1946$ - 2016) of the Russian Academic of Sciences. 